\title{
MAPPINGS OF FINITE DISTORTION: REVERSE INEQUALITIES FOR THE JACOBIAN
}

\author{
STANISLAV HENCL, PEKKA KOSKELA AND XIAO ZHONG
}

\begin{abstract}
Let $f$ be a non-constant mapping of finite distortion. We establish integrability results on $1 / J_{f}$ by studying weights that satisfy a weak reverse Hölder inequality where the associated constant can depend on the ball in question. Here $J_{f}$ is the Jacobian determinant of $f$.
\end{abstract}

\section{INTRODUCTION}

In this paper, we consider the class of mappings of finite distortion. Let $\Omega$ be a domain in $\mathbb{R}^{n}(n \geq 2)$. We say that a mapping $f: \Omega \rightarrow \mathbb{R}^{n}$ has finite distortion if $f$ belongs to the Sobolev space $W_{\text {loc }}^{1,1}\left(\Omega, \mathbb{R}^{n}\right)$; the Jacobian determinant $J_{f}=\operatorname{det}(D f)$ of $f$ ( $D f$ being the differential matrix of $f$ ) is locally integrable in $\Omega$, and there is a measurable function $K(x) \geq 1$, finite almost everywhere, such that $f$ satisfies the distortion inequality

$$
|D f(x)|^{n} \leq K(x) J_{f}(x), \quad \text { for almost every } x \text { in } \Omega .
$$

Notice that when $K(x)$ is bounded, the above conditions imply that the differential of $f$ is locally $n$-integrable, and $f$ is a mapping of bounded distortion in the sense of Reshetnyak [31], or a quasiregular mapping [32]. These mappings are continuous (i.e. have a continuous representative) and either constant or both discrete and open. This means that the pre-image of a point cannot accumulate in $\Omega$ and that $f$ maps open sets to open sets. All these three properties may fail for a general mapping of finite distortion, but sharp integrability criteria for the validity of these properties have recently been established, see [4], [10], [11], [14], [18], [19], [21], [24], [25], [26], [28]. In the setting considered in this paper our mapping will have all these three properties.

The distortion inequality implies that $J_{f}(x) \geq 0$ almost everywhere, and one can give examples where $J_{f}$ vanishes in a set of positive measure for a non-constant mapping. Very recently, Koskela and Malý [27] proved, under sharp integrability conditions on $K$, that, unless $f$ is constant, $J_{f}(x)>0$ almost everywhere. The conditions are the same as for discreteness and openness.

2000 Mathematics Subject Classification. 30C65, 26B10.

Key words and phrases. Mapping of finite distortion, Jacobian, reverse inequalities, weights. 
In the case of quasiregular mappings, a much stronger statement holds. Indeed, relying on the fundamental work of Gehring [9], Elcrat and Meyers [5] and Martio [29] proved the reverse Hölder inequality

$$
\left(\frac{1}{|B|} \int_{B} J_{f}^{p} d x\right)^{1 / p} \leq C \frac{1}{|B|} \int_{B} J_{f} d x
$$

for the Jacobian of a quasiregular mapping $f$. Here $B \subset \Omega^{\prime} \subset \subset \Omega$, is a ball and the constant $C$ will in general depend on $\Omega^{\prime}$ and on the behavior of $f$ on $\Omega^{\prime}$ (the maximal multiplicity $N\left(f, \Omega^{\prime}\right)$ ), and $p>1$ depends on $n$ and the $L^{\infty}$-norm of $K(x)$. Thus $J_{f}$ is (locally) a Muckenhoupt $A_{\infty}$-weight [30], and so

$$
J_{f}^{-\varepsilon} \in L_{\mathrm{loc}}^{1}\left(\Omega^{\prime}\right)
$$

for some $\varepsilon>0$, see [3].

One is then motivated to consider the case of mappings of finite distortion, and to prove some kind of an integrability result on $J_{f}^{-1}$, based on a suitable reverse inequality of the Jacobian. To this end, let $f \in W_{\text {loc }}^{1,1}\left(\Omega, \mathbb{R}^{n}\right)$ be a mapping with exponentially integrable distortion $K(x)$, that is, $\exp (\beta K) \in L_{\text {loc }}^{1}(\Omega)$ for some $\beta>0$. Then $f$ is continuous [18], and either constant or both discrete and open [24]. Let us recall a higher integrability result from [6, Theorem 1.1] (see [1], [16], [17] for the earlier results)

$$
f_{B} J_{f} \log ^{\alpha}\left(e+\frac{J_{f}}{f_{2 B} J_{f}}\right) d x \leq C\left(f_{2 B} \exp (\beta K)\right) f_{2 B} J_{f}
$$

for every ball $B$ such that $2 B \subset \subset \Omega$. Invoking the doubling property of the Jacobian of a mapping of finite distortion (see Lemma 5.2) and using the Jensen inequality we conclude that

$$
f_{B} J_{f} \log ^{\alpha}\left(e+\frac{J_{f}}{f_{4 B} F f_{B} J_{f}}\right) \leq\left(f_{4 B} F\right)^{2} f_{B} J_{f},
$$

where $F=C \exp (C K)$ is an integrable function. Notice that (1.1) is sort of a reverse inequality. If the function $F$ is bounded, then (1.1) implies that $J_{f}$ is an $A_{\infty}$-weight [8], and thus $J_{f}^{-\varepsilon}$ is locally integrable for some $\varepsilon>0$. Of course, by our assumption on the distortion function $K(x)$, we merely have that $F$ is integrable. This leads us to the study of non-negative weights that satisfy a reverse inequality such as (1.1), where the constant is allowed to depend on the ball in question. For the weights as above, we will prove that there is $\gamma>0$ such that

$$
\log ^{\gamma} \log \left(e+1 / J_{f}\right) \in L_{\text {loc }}^{1},
$$

see Theorem 3.8. This is an essentially sharp conclusion for such a weight, and thus no better conclusion can be drawn from the (sharp) inequality (1.1).

We expect that radial mappings exhibit the extreme integrability properties of $J_{f}^{-1}$. The analysis of such mappings (see Section 6 below) indicates that (1.2) is far from optimal. We are thus led to look 
for reverse inequalities different from (1.1). We establish the reverse inequality

$$
f_{B} J_{f} \leq c\left(f_{2 B} F\right)^{t}\left(f_{B} J_{f}^{q}\right)^{\frac{1}{q}}
$$

where $F \in L_{\text {loc }}^{1}, t>0$ and $0<q<1$, and a certain variant of it. We again analyze the corresponding weights and prove essentially optimal results for them.

The following theorem gives our contribution to the integrability of $1 / J_{f}$ under the exponential integrability assumption on $K$.

Theorem 1.1. Let $\Omega \subset \mathbb{R}^{n}$ be a domain and let $f \in W_{\mathrm{loc}}^{1,1}\left(\Omega, \mathbb{R}^{n}\right)$ be a mapping of finite distortion.

(i) Suppose that $n \geq 3$ and that there is $\beta>0$ such that $\exp (\beta K) \in$ $L_{\mathrm{loc}}^{1}(\Omega)$. Then there is $s=C(n)>1$ such that

$$
\exp \left(\log ^{s} \log \left(e+\frac{1}{J_{f}(x)}\right)\right) \in L_{\mathrm{loc}}^{1}(\Omega) .
$$

(ii) Suppose that $n=2, \gamma>0$ and let $\Omega^{\prime} \subset \subset \Omega$ be a fixed open set. Then there is a finite constant $\beta=C\left(\gamma, N\left(f, \Omega^{\prime}\right)\right)>0$ such that if $\exp (\beta K) \in L^{1}\left(\Omega^{\prime}\right)$ then

$$
\log ^{\gamma}\left(e+\frac{1}{J_{f}(x)}\right) \in L_{\mathrm{loc}}^{1}\left(\Omega^{\prime}\right)
$$

It seems likely that Theorem 1.1 allows for an analog, where the exponential integrability is slightly relaxed to the conditions in [26]. We would like to see such an improvement.

If we a priori assume, say, that $f \in W_{\text {loc }}^{1, n}\left(\Omega, \mathbb{R}^{n}\right)$, then already the $p$-integrability of its distortion function $K(x)$ for some $p>n-1 \mathrm{im}$ plies discreteness and openness for a non-constant mapping. Thus one expects for a version of Theorem 1.1 under such assumptions. Our techniques also work in this case and give the following result.

Theorem 1.2. Let $\Omega \subset \mathbb{R}^{n}$ be a domain and let $\varepsilon>0$. Suppose that $f \in W_{\text {loc }}^{1, n}\left(\Omega, \mathbb{R}^{n}\right)$ is a mapping of finite distortion and suppose that $K \in L_{\mathrm{loc}}^{n-1+\varepsilon}(\Omega)$. Then there is $\gamma=\gamma(n)>0$ such that

$$
\log ^{\gamma} \log \left(e+\frac{1}{J_{f}(x)}\right) \in L_{\mathrm{loc}}^{1}(\Omega) .
$$

As discussed above, the conclusions we draw from the reverse inequalities are essentially optimal. However, the integrability results on $1 / J_{f}$ themselves do not seem to be best possible. It remains to be seen if new a priori inequalities that would result on better integrability conclusions can be found.

The paper is organized as follows. In Section 2 we introduce the necessary notation. Section 3 deals with the integrability of non-negative weights that arise from our approach. We believe that these weights 
may have their own interests. In Section 4 we show by means of examples that the results from Section 3 are sharp. We continue by applying the integrability results in Section 5 to mappings of finite distortion. Finally, in Section 6, we discuss the optimality of the results for mappings of finite distortion.

\section{Preliminaries}

2.1. Notation. We write $\|x\|$ for the Euclidean norm of $x \in \mathbb{R}^{n}$ and denote the Lebesgue measure of a measurable set $E \subset \mathbb{R}^{n}$ by $|E|$. By $\Omega$ we will always mean a domain in $\mathbb{R}^{n}, n \geq 2$, i.e. connected open set. Given two functions $F, G: \Omega \rightarrow \mathbb{R}$ we write $G \sim F$ if there is a constant $C \geq 1$ such that $F(x) / C \leq G(x) \leq C F(x)$ for every $x \in \Omega$.

Let $A \subset \mathbb{R}^{n}$ be a measurable set and $f \in L^{1}(A)$. The average integral will be denoted by

$$
f_{A} f=\frac{1}{|A|} \int_{A} f
$$

We say that $x \in \Omega$ is a Lebesgue point of $f \in L_{\text {loc }}^{1}(\Omega)$ if

$$
\lim _{r \rightarrow 0} \sup _{c: x \in B(c, r)}\left|f(x)-f_{B(c, r)} f\right|=0 .
$$

It is well-known that almost every point is a Lebesgue point.

We use the convention that $C$ denotes some positive constant where the value of this constant may differ from occur to occur as usual. We write e.g. $C(a, b)$ to indicate an absolute constant for fixed $a$ and $b$.

For the convenience of the reader we state here the standard covering theorem that that we will later employ.

Theorem 2.1 (Vitali). Let $\mathcal{B}$ be an arbitrary family of balls in $\mathbb{R}^{n}$ with

$$
\sup \{\operatorname{diam} B: B \in \mathcal{B}\}<\infty .
$$

Then there exists a disjoint (at most countable) subfamily $\left\{B\left(x_{i}, r_{i}\right)\right\} \subset$ $\mathcal{B}$ such that

$$
\bigcup_{B \in \mathcal{B}} B \subset \bigcup B\left(x_{i}, 5 r_{i}\right)
$$

We say that the distributional Jacobian of $f=\left(f_{1}, \ldots, f_{n}\right): \Omega \rightarrow \mathbb{R}^{n}$ is equal to the point-wise Jacobian if $f \in W^{1,1}\left(\Omega, \mathbb{R}^{n}\right)$ and for every test function $\phi \in C_{0}^{\infty}(\Omega)$ we have

$$
\int_{\Omega} \phi J\left(x,\left(f_{1}, f_{2}, \ldots, f_{n}\right)\right) d x=-\int_{\Omega} f_{1} J\left(x,\left(\phi, f_{2}, \ldots, f_{n}\right)\right) .
$$

2.2. Area formula. We say that a mapping $f: \Omega \rightarrow \mathbb{R}^{n}$ satisfies the Lusin condition $N$ if the implication $|E|=0 \Longrightarrow|f(E)|=0$ holds for any measurable set $E \subset \Omega$.

We continue with the well-known area formula. Let $f \in W_{\text {loc }}^{1,1}\left(\Omega ; \mathbb{R}^{n}\right)$ and let $\eta$ be a nonnegative Borel measurable function on $\mathbb{R}^{n}$. The 
multiplicity function $N(f, \Omega, y)$ of $f$ is defined as the number of preimages of $y$ under $f$ in $\Omega$. Without any additional assumption we have

$$
\int_{\Omega} \eta(f(x))\left|J_{f}(x)\right| d x \leq \int_{\mathbb{R}^{n}} \eta(y) N(f, \Omega, y) d y .
$$

This follows from the area formula for Lipschitz mappings and from the fact that $\Omega$ can be exhausted up to a set of measure zero by sets the restriction to which of $f$ is Lipschitz continuous (see [7]). For the converse inequality

$$
\int_{\mathbb{R}^{n}} \eta(y) N(f, \Omega, y) d y \leq \int_{\Omega} \eta(f(x))\left|J_{f}\right| d x
$$

the Lusin condition $N$ should be assumed, the reason for validity is the same as that for (2.2).

2.3. Topological properties. A mapping $f: \Omega \rightarrow \mathbb{R}^{n}$ is said to be discrete if the number of the pre-images of any point of $\mathbb{R}^{n}$ is locally finite in $\Omega$, and open if image of every open set is an open set.

If $f: \Omega \rightarrow \mathbb{R}^{n}$ is continuous, open and discrete and $A \subset \Omega$ is a compact set then

$$
N(f, A):=\sup _{y \in \mathbb{R}^{n}} N(f, A, y)<\infty
$$

(see [32, I. Proposition $4.10(3)]$ ).

2.4. Capacity. For disjoint compact sets $E$ and $F$ in a domain $U \subset$ $\mathbb{R}^{n}$, we define the conformal capacity of the pair $(E, F)$ by

$$
\operatorname{cap}(E, F ; U)=\inf _{u} \int_{U}|\nabla u(x)|^{n} d x
$$

where the infimum is taken over all functions $u \in C^{\infty}(U)$ which satisfy $u(x) \leq 0$ for $x \in E$ and $u(x) \geq 1$ for $x \in F$.

We will need the following fundamental property of conformal capacity. Suppose that $E$ and $F$ are connected compact sets such that $\operatorname{diam}(E)<\operatorname{dist}(E, F)$, then

$$
\operatorname{cap}\left(E, F ; \mathbb{R}^{n}\right) \leq C(n)\left(\log \frac{\operatorname{dist}(E, F)}{\operatorname{diam} E}\right)^{1-n}
$$

(for this see e.g. [15, Fact 3.1(e)]).

We say that a set $\tilde{E} \subset \mathbb{R}^{n}$ joins two sets $A, B \subset \mathbb{R}^{n}$ if there is a connected subset of $\tilde{E}$ which intersects both $A$ and $B$. The following lemma is standard (see e.g. [13, Theorem 5.9]).

Lemma 2.2. Let $\tilde{E}$ be a connected compact set joining a point a $\in$ $\mathbb{R}^{n}$ to the sphere $S(a, r)$ and $\tilde{F}$ be a connected compact set joining the sphere $S(a, 2 r)$ to $S(a, 4 r)$. Suppose that $u \in W^{1,1}(B(0,4 r))$ is 
continuous and satisfies $u \geq 0$ on $\tilde{E}$ and $u \leq 1$ on $\tilde{F}$. Then for all $n-1<p \leq n$

$$
\int_{B(a, 4 r)}|\nabla u|^{p} \geq C(p, n) r^{n-p}
$$

\section{Weighted $A_{\infty}$ COnditions}

Let $\Omega \subset \mathbb{R}^{n}$ be a domain, $p>1, t>0, \alpha>0$ and let $F \in L_{\text {loc }}^{1}(\Omega)$. Motivated by the applications in mappings of finite distortion we study three classes of non-negative weights $w \in L_{\text {loc }}^{1}(\Omega)$. The first class consists of weights which satisfy

$$
\left(f_{B} w^{p}\right)^{1 / p} \leq\left(f_{2 B} F\right)^{t} f_{B} w
$$

for every ball $B$ such that $2 B \subset \Omega$. Weights from the second class satisfy

$$
\left(f_{B} w^{p}\right)^{1 / p} \leq \exp \left(\left(f_{2 B} F\right)^{t}\right) f_{B} w
$$

for every ball $B$ such that $2 B \subset \Omega$. Finally, weights from the third class satisfy

$$
f_{B} \frac{w}{f_{B} w} \log ^{\alpha}\left(e+\frac{w}{f_{4 B} F f_{B} w}\right) \leq\left(f_{4 B} F\right)^{2}
$$

for every ball $B$ such that $4 B \subset \Omega$. Without loss of generality, we will suppose that $F \geq 1$ almost everywhere and therefore $f_{2 B} F \geq 1$. Let us note that the fact that $F$ is integrated over $2 B$ (or $4 B$ ) is not important. In fact, given any fixed constant $s \geq 1$, we can replace $f_{2 B} F$ by $f_{s B} F$ and with obvious minor modifications we can obtain results similar to those presented below.

Note that if $F$ is a constant function, then our weights satisfy a reverse Hölder inequality and therefore they belong to the usual Muckenhaupt class $A_{\infty}$ (see [3] and [2]).

Under each of these three conditions we will be able to obtain some integrability results for the function $\frac{1}{w(x)}$. Since the proofs are quite similar we will give the details only in the first case and then simply point out the differences in the other cases.

We start with the following easy lemma.

Lemma 3.1. Let $\Omega \subset \mathbb{R}^{n}$ be a domain, $p>1, t>0$ and let $F \in$ $L_{\mathrm{loc}}^{1}(\Omega)$. Suppose that a non-negative weight $w \in L_{\mathrm{loc}}^{1}(\Omega)$ satisfies (3.1) for every ball $B$ such that $2 B \subset \Omega$. Set

$$
a(B)=\left(2\left(f_{2 B} F\right)^{p t}\right)^{1 /(p-1)} .
$$


Then for every ball $B$ such that $2 B \subset \Omega$ we have

$$
\int_{B} w \leq 2 \int_{\left\{x \in B: w(x)<a(B) f_{B} w\right\}} w .
$$

Proof. Set $\tilde{w}(x)=w(x) / f_{B} w$. We will prove the statement by contradiction. Suppose that there is a ball $B$ such that

$$
\int_{B} w>2 \int_{\{x \in B: \tilde{w}(x)<a(B)\}} w
$$

Clearly

$$
\int_{\{\tilde{w} \geq a(B)\}} \tilde{w}=\int_{B} \tilde{w}-\int_{\{\tilde{w}<a(B)\}} \tilde{w}>|B|-\frac{|B|}{2}=\frac{|B|}{2} .
$$

Thanks to (3.4) and (3.6) we have

$f_{B} \tilde{w}^{p} \geq \frac{1}{|B|} \int_{\{\tilde{w} \geq a(B)\}} \tilde{w} \tilde{w}^{p-1} \geq 2\left(f_{2 B} F\right)^{p t} \frac{1}{|B|} \int_{\{\tilde{w} \geq a(B)\}} \tilde{w}>\left(f_{2 B} F\right)^{p t}$

which gives us a contradiction to (3.1).

Lemma 3.2. Let $\Omega \subset \mathbb{R}^{n}$ be a domain, $p>1, t>0$ and let $F \in$ $L_{\mathrm{loc}}^{1}(\Omega)$. Suppose that a non-negative weight $w \in L_{\mathrm{loc}}^{1}(\Omega)$ satisfies (3.2) for every ball $B$ such that $2 B \subset \Omega$. Set

$$
a(B)=\left(2 \exp \left(p\left(f_{2 B} F\right)^{t}\right)\right)^{1 /(p-1)} .
$$

Then for every ball $B$ such that $2 B \subset \Omega$ we have

$$
\int_{B} w \leq 2 \int_{\left\{x \in B: w(x)<a(B) f_{B} w\right\}} w .
$$

Proof. Supposing that (3.8) is not true we obtain (3.6) (of course $a(B)$ is now given by (3.7)). Thanks to (3.7) and (3.6) we have

$$
f_{B} \tilde{w}^{p} \geq 2 \exp \left(p\left(f_{2 B} F\right)^{t}\right) \frac{1}{|B|} \int_{\{\tilde{w} \geq a(B)\}} \tilde{w}>\exp \left(p\left(f_{2 B} F\right)^{t}\right)
$$

which gives us a contradiction with (3.2).

Lemma 3.3. Let $\Omega \subset \mathbb{R}^{n}$ be a domain, $\alpha>0$ and let $F \in L_{\mathrm{loc}}^{1}(\Omega)$. Suppose that a non-negative weight $w \in L_{\mathrm{loc}}^{1}(\Omega)$ satisfies (3.3) for every ball $B$ such that $4 B \subset \Omega$. Set

$$
a(B)=f_{4 B} F \exp \left(\left(2\left(f_{4 B} F\right)^{2}\right)^{1 / \alpha}\right) .
$$

Then for every ball $B$ such that $4 B \subset \Omega$ we have

$$
\int_{B} w \leq 2 \int_{\left\{x \in B: w(x)<a(B) f_{B} w\right\}} w .
$$


Proof. Supposing that (3.10) is not true we obtain $(3.6)(a(B)$ is now given by (3.9)). Therefore (3.9) and (3.6) give us

$$
\begin{gathered}
f_{B} \tilde{w} \log ^{\alpha}\left(e+\frac{\tilde{w}}{f_{4 B} F}\right) \geq \frac{1}{|B|} \int_{\{\tilde{w} \geq a(B)\}} \tilde{w} \log ^{\alpha}\left(e+\frac{\tilde{w}}{f_{4 B} F}\right) \geq \\
\geq 2\left(f_{4 B} F\right)^{2} \frac{1}{|B|} \int_{\{\tilde{w} \geq a(B)\}} \tilde{w}>\left(f_{4 B} F\right)^{2}
\end{gathered}
$$

which gives us a contradiction with (3.3).

Our goal is to estimate integrals of $1 / w$. The following lemma will allow us to make the technically convenient assumption that $w$ be bounded away from zero. It is easy to see that analogous statements under conditions (3.2) and (3.3) are also valid.

Lemma 3.4. Let $\Omega \subset \mathbb{R}^{n}$ be a domain, $p>1, t>0$ and let $F \in$ $L_{\mathrm{loc}}^{1}(\Omega)$. Suppose that a non-negative weight $w \in L_{\mathrm{loc}}^{1}(\Omega)$ satisfies (3.1) for every ball $B$ such that $2 B \subset \Omega$. Then there is a locally integrable function $\tilde{F}$ such that for every $\delta>0$ the function $w_{\delta}:=\max \{\delta, w\}$ satisfies condition (3.1) with $\tilde{F}$ (i.e., $\tilde{F}$ does not depend on $\delta$ ).

Proof. Fix a ball $B$. Clearly $f_{B} w_{\delta} \geq \delta$ and $f_{B} w_{\delta} \geq f_{B} w$. Therefore (3.1) and $f_{2 B} F \geq 1$ imply

$$
\begin{gathered}
\int_{B}\left(\frac{w_{\delta}}{f w_{\delta}}\right)^{p} \leq \int_{\{x \in B: w(x)>\delta\}}\left(\frac{w}{f w}\right)^{p}+\int_{\{x \in B: w(x) \leq \delta\}}\left(\frac{\delta}{\delta}\right)^{p} \leq \\
\leq|B|\left(f_{2 B} F\right)^{p t}+|B| \leq 2|B|\left(f_{2 B} F\right)^{p t} .
\end{gathered}
$$

Inspired by [3, Theorem IV and Theorem V], we establish the following results on the integrability of $\frac{1}{w(x)}$.

Theorem 3.5. Let $\Omega \subset \mathbb{R}^{n}$ be a domain, $p>1, t>0$ and let $F \in$ $L_{\mathrm{loc}}^{1}(\Omega)$. Suppose that a non-negative weight $w \in L_{\mathrm{loc}}^{1}(\Omega)$ satisfies (3.1) for every ball $B$ such that $2 B \subset \Omega$. Then for every $\varepsilon>0$ we have

$$
\log ^{\frac{p-1}{p t}-\varepsilon}\left(e+\frac{1}{w(x)}\right) \in L_{\mathrm{loc}}^{1}(\Omega) .
$$

Proof. First we will assume that there is $\delta>0$ such that $w \geq \delta$. Set

$$
q=\frac{p-1}{p t}, \beta=1-q+\varepsilon, A(\lambda)=\log ^{1-\frac{\varepsilon}{2 q}} \lambda \text { and } B(\lambda)=\frac{1}{\lambda \log ^{\beta} \lambda} .
$$

Clearly we can assume that $\varepsilon$ is so small that $\beta<1-\frac{\varepsilon}{2 q}$.

Fix a ball $B$ such that $2 B \subset \subset \Omega$ and fix $\lambda>\max \left\{1 / f_{B} w, 2\right\}$. We claim that

$$
|\{x \in B: 1 / w(x)>\lambda\}| \leq \frac{C}{A(\lambda)^{q}}+C \lambda \int_{\left\{x \in B: \frac{\lambda}{A(\lambda)}<\frac{1}{w(x)}\right\}} w .
$$


To this end, denote $S=\{x \in B: 1 / w(x)>\lambda\}$ and let $\tilde{S}$ be the set of Lebesgue points of $w$ in $S$ (i.e., $|S \backslash \tilde{S}|=0$ ). For every $s \in \tilde{S}$ we have

$$
\lim _{r \rightarrow 0} \sup _{c: s \in B(c, r)}\left|w(s)-f_{B(c, r)} w\right|=0 .
$$

Since $w(s)<1 / \lambda<f_{B} w$ it follows that it is possible to find a ball $B_{s} \subset B$ such that

$$
\lambda<\frac{\left|B_{s}\right|}{\int_{B_{s}} w} \leq 2 \lambda .
$$

We can use Vitali covering theorem (see Theorem 2.1) for the family $\left\{2 B_{s}\right\}_{s \in \tilde{S}}$ to obtain balls $B_{i} \subset B$ such that

$$
\begin{gathered}
\tilde{S} \subset \bigcup_{i} 10 B_{i} \text { and }|\{x \in B: 1 / w(x)>\lambda\} \backslash \tilde{S}|=0, \\
\lambda<\frac{\left|B_{i}\right|}{\int_{B_{i}} w} \leq 2 \lambda \text { for every } i \text { and }
\end{gathered}
$$

the balls $2 B_{i}$ are pairwise disjoint.

We will use notation $a(B)$ as in (3.4). Assuming that $\tilde{B}$ is a ball such that $2 \tilde{B} \subset \Omega$ and $A(\lambda) \leq a(\tilde{B})$ we obtain

$$
|\tilde{B}| \leq \frac{C(p, t, n) \int_{2 \tilde{B}} F}{A(\lambda)^{q}} .
$$

From (3.13), (3.16), (3.14), (3.15) and (3.5) we have

$$
\begin{gathered}
|\{x \in B: 1 / w(x)>\lambda\}| \leq C(n) \sum_{i}\left|B_{i}\right| \leq \\
\leq C(n) \sum_{i: a\left(B_{i}\right) \geq A(\lambda)}\left|B_{i}\right|+C(n) \sum_{i: a\left(B_{i}\right)<A(\lambda)}\left|B_{i}\right| \leq \\
\leq \frac{C(p, t, n)}{A(\lambda)^{q}} \sum_{i} \int_{2 B_{i}} F+2 C(n) \lambda \sum_{i: a\left(B_{i}\right)<A(\lambda)} \int_{B_{i}} w \leq \\
\leq \frac{C(p, t, n)}{A(\lambda)^{q}} \int_{2 B} F+C(n) \lambda \sum_{i: a\left(B_{i}\right)<A(\lambda)} \int_{\left\{x \in B_{i}: w(x)<a\left(B_{i}\right) f_{B_{i}} w\right\}} w \leq \\
\leq \frac{C(p, t, n, F)}{A(\lambda)^{q}}+C(n) \lambda \int_{\left\{x \in B: \frac{\lambda}{A(\lambda)}<\frac{1}{w(x)}\right\}} w,
\end{gathered}
$$

which proves (3.12).

Denote $E=\max \left\{1 / f_{B} w, 2\right\}$. We multiply both sides of inequality (3.12) by $B(\lambda)$ and integrate with respect to $\lambda$ from $E$ to $\infty$ and obtain that

$$
\int_{E}^{\infty} B(\lambda)|\{x \in B: 1 / w(x)>\lambda\}| d \lambda \leq
$$




$$
\leq C(p, t, n, F) \int_{E}^{\infty} \frac{B(\lambda)}{A(\lambda)^{q}}+C(n) \int_{E}^{\infty} B(\lambda) \lambda \int_{\left\{x \in B: \frac{\lambda}{A(\lambda)}<\frac{1}{w(x)}\right\}} w .
$$

Set $w_{1}(x)=1 / w(x)$ and $d \mu_{1}(x)=w(x) d x$.

We can estimate the left-hand side of inequality (3.17) by

$$
\int_{E}^{\infty} \frac{1}{\lambda \log ^{\beta} \lambda}\left|\left\{x \in B: w_{1}(x)>\lambda\right\}\right| d \lambda \geq
$$

$$
\begin{gathered}
\geq C(\beta) \int_{\left\{x \in B: w_{1}(x)>E\right\}} \log ^{1-\beta}\left(e+w_{1}(x)\right) d x-C(\beta)|B| \log ^{1-\beta}(e+E)= \\
=C(q, \varepsilon) \int_{\{x \in B: 1 / w(x)>E\}} \log ^{q-\varepsilon}\left(e+\frac{1}{w(x)}\right) d x-C(q, \varepsilon,|B|, E) .
\end{gathered}
$$

Since $\beta+q-\frac{\varepsilon}{2}>1$ we have

$$
\int_{E}^{\infty} \frac{B(\lambda)}{A(\lambda)^{q}} d \lambda=\int_{E}^{\infty} \frac{d \lambda}{\lambda \log ^{\beta+q-\frac{\varepsilon}{2}} \lambda}<\infty
$$

We use substitution $\tilde{\lambda}=\frac{\lambda}{A(\lambda)}$ (i.e., $\lambda \sim \tilde{\lambda} \log ^{1-\frac{\varepsilon}{2 q}} \tilde{\lambda}$ ) in the second term on the right-hand side of (3.17) to obtain

$$
\begin{gathered}
\int_{E}^{\infty} B(\lambda) \lambda \mu_{1}\left(\left\{x \in B: w_{1}>\frac{\lambda}{A(\lambda)}\right\}\right) d \lambda \leq \\
\leq C(\beta) \int_{\frac{E}{A(E)}}^{\infty} \frac{1}{\log ^{\beta} \tilde{\lambda}} \mu_{1}\left(\left\{x \in B: w_{1}>\tilde{\lambda}\right\}\right) \log ^{1-\frac{\varepsilon}{2 q}} \tilde{\lambda} d \tilde{\lambda} \leq \\
\leq C(q, \varepsilon) \int_{\left\{x \in B: w_{1}(x)>\frac{E}{A(E)}\right\}} w_{1}(x) \log ^{1-\beta-\frac{\varepsilon}{2 q}} w_{1}(x) d \mu_{1}(x) \leq \\
\leq C(q, \varepsilon) \int_{\left\{x \in B: 1 / w(x)>\frac{E}{A(E)}\right\}} \log ^{q-\varepsilon-\frac{\varepsilon}{2 q}}\left(e+\frac{1}{w(x)}\right) d x .
\end{gathered}
$$

Clearly we can find a constant $M$ big enough such that $M>E$ and $\log ^{-\frac{\varepsilon}{2 q}}(e+M)$ is as small as we wish. Combining this with (3.17), (3.18), (3.19) and (3.20) we arrive at

$$
\begin{gathered}
\int_{\{x \in B: 1 / w(x)>E\}} \log ^{q-\varepsilon}\left(e+\frac{1}{w(x)}\right) d x \leq C(p, t, \varepsilon, n, E,|B|, F)+ \\
+C(q, \varepsilon) \int_{\left\{x \in B: 1 / w(x)>\frac{E}{A(E)}\right\}} \log ^{q-\varepsilon-\frac{\varepsilon}{2 q}}\left(e+\frac{1}{w(x)}\right) d x \\
\leq C(p, t, \varepsilon, n, E,|B|, F)+\frac{1}{2} \int_{\{x \in B: 1 / w(x)>M\}} \log ^{q-\varepsilon}\left(e+\frac{1}{w(x)}\right) d x .
\end{gathered}
$$

Since $w \geq \delta$ for some $\delta>0$, all the integrals are finite and we obtain $(3.21)$

$$
\int_{\{x \in B: 1 / w(x)>M\}} \log ^{q-\varepsilon}\left(e+\frac{1}{w(x)}\right) d x \leq C\left(p, t, n, \varepsilon,|B|, \int_{B} w, \int_{2 B} F\right)
$$

which implies (3.11). 
Now suppose that $w \in L_{\mathrm{loc}}^{1}(\Omega)$ is arbitrary non-negative weight. Thanks to Lemma 3.4 we have that (3.21) holds for $w_{\delta}=\max \{w, \delta\}$ and it is easy to check that we can make the constants $C$ and $M$ independent on the value of $\delta$ if $\delta$ is sufficiently small. Sending $\delta$ to 0 we obtain the result also for $w$.

Remark 3.6. We will need a more explicit form of the constant from (3.21) later. Namely, if $t \leq 1$ and $\varepsilon=\frac{1}{3} \frac{p-1}{p t}$, by a careful analysis of the constants in the proof of Theorem 3.5, we otain the estimate

$$
\int_{B} \log ^{\frac{2}{3} \frac{p-1}{p t}}\left(e+\frac{1}{w(x)}\right) d x \leq C\left(p, n,|B|, \int_{B} w\right)^{\frac{1}{t}} \int_{2 B} F .
$$

The following two theorems give the integrability result of $1 / w(x)$ for the weights $w(x)$ satisfying (3.2) and (3.3), respectively.

Theorem 3.7. Let $\Omega \subset \mathbb{R}^{n}$ be a domain, $p>1, t>0$ and let $F \in$ $L_{\mathrm{loc}}^{1}(\Omega)$. Suppose that a non-negative weight $w \in L_{\mathrm{loc}}^{1}(\Omega)$ satisfies (3.2) for every ball $B$ such that $2 B \subset \Omega$. Then for every $\varepsilon>0$ we have

$$
\log ^{\frac{1}{t}-\varepsilon} \log \left(e+\frac{1}{w(x)}\right) \in L_{\text {loc }}^{1}(\Omega) .
$$

Proof. The proof of this theorem is similar to the proof of Theorem 3.5 and therefore we will not give all the details. Fix a ball $B$ such that $2 B \subset \subset \Omega$ and fix $\lambda>\max \left\{1 / f_{B} w, 2\right\}$. Set

$$
\beta=1-\frac{1}{t}+\varepsilon, A(\lambda)=\log \lambda \log ^{1-\varepsilon} \log \lambda \text { and } B(\lambda)=\frac{1}{\lambda \log \lambda \log ^{\beta} \log \lambda} .
$$

Clearly we may assume that $\varepsilon$ is so small that $\varepsilon<\frac{1}{2 t}$. Since $a(B)$ is now given by (3.7) we obtain analogously to the proof of (3.17) that

$$
\begin{gathered}
\int_{E}^{\infty} B(\lambda)|\{x \in B: 1 / w(x)>\lambda\}| d \lambda \leq \\
\leq C(p, t, n, F) \int_{E}^{\infty} \frac{B(\lambda)}{\log ^{1 / t} A(\lambda)}+C(n) \int_{E}^{\infty} B(\lambda) \lambda \int_{\left\{x \in B: \frac{\lambda}{A(\lambda)}<\frac{1}{w(x)}\right\}} w .
\end{gathered}
$$

From $1-1 / t<\beta$ we obtain that the first integral on the right hand side is finite. Similarly to (3.20) we use substitution $\tilde{\lambda}=\frac{\lambda}{A(\lambda)}$ (i.e., $\left.\lambda \sim \tilde{\lambda} \log \tilde{\lambda} \log ^{1-\varepsilon} \log \tilde{\lambda}\right)$ in the second term on the right hand side to obtain

$$
\begin{gathered}
\int_{E}^{\infty} B(\lambda) \lambda \mu_{1}\left(\left\{x \in B: w_{1}>\frac{\lambda}{A(\lambda)}\right\}\right) d \lambda \leq \\
\leq C(p, t, \varepsilon) \int_{\left\{x \in B: 1 / w(x)>\frac{E}{A(E)}\right\}} \log ^{\frac{1}{t}-2 \varepsilon} \log \left(e+\frac{1}{w(x)}\right) d x .
\end{gathered}
$$

We can find $M$ big enough to make $\log ^{-\varepsilon} \log (e+M)$ very small and therefore analogously to the proof of (3.21) we obtain

$$
\int_{\{x \in B: 1 / w(x)>M\}} \log ^{\frac{1}{t}-\varepsilon} \log \left(e+\frac{1}{w(x)}\right) d x \leq C\left(p, t, n, \varepsilon,|B|, \int_{B} w, \int_{2 B} F\right)
$$


for weights which satisfy $w \geq \delta$ for some $\delta>0$. Using an analog of Lemma 3.4 for weights satisfying (3.2) we obtain the result also for general weights.

Theorem 3.8. Let $\Omega \subset \mathbb{R}^{n}$ be a domain, $\alpha>0$ and let $F \in L_{\text {loc }}^{1}(\Omega)$. Suppose that a non-negative weight $w \in L_{\text {loc }}^{1}(\Omega)$ satisfies (3.3) for every ball $B$ such that $4 B \subset \Omega$. Then for every $\varepsilon>0$ we have

$$
\log ^{\frac{\alpha}{2}-\varepsilon} \log \left(e+\frac{1}{w(x)}\right) \in L_{\mathrm{loc}}^{1}(\Omega)
$$

Proof. Fix a ball $B$ such that $4 B \subset \subset \Omega$ and fix $\lambda>\max \left\{1 / f_{B} w, 2\right\}$. Set

$\beta=1-\frac{\alpha}{2}+\varepsilon, A(\lambda)=\log \lambda \log ^{1-\varepsilon} \log \lambda$ and $B(\lambda)=\frac{1}{\lambda \log \lambda \log ^{\beta} \log \lambda}$.

Clearly we may assume that $\varepsilon$ is so small that $\varepsilon<\frac{\alpha}{4}$. Since $a(B)$ is now given by (3.9) we obtain analogously to the proof of (3.17) that

$$
\begin{gathered}
\int_{E}^{\infty} B(\lambda)|\{x \in B: 1 / w(x)>\lambda\}| d \lambda \leq \\
\leq C(\alpha, \varepsilon, n, F) \int_{E}^{\infty} \frac{B(\lambda)}{\log ^{\alpha / 2} A(\lambda)}+C(n) \int_{E}^{\infty} B(\lambda) \lambda \int_{\left\{x \in B: \frac{\lambda}{A(\lambda)}<\frac{1}{w(x)}\right\}} w .
\end{gathered}
$$

Since an analogof Lemma 3.4 is true for weights satisfying (3.3) we obtain the result analogously to the proof of Theorem 3.7.

\section{EXAMPLES OF WEIGHTS}

The examples in this section show that the results on the integrability of $\frac{1}{w(x)}$ from the last section cannot be essentially improved. Again we give all the details and computations only in the first case. For simplicity, we give the examples in the real line; similar examples can be constructed in higher dimensions.

Example 4.1. Let $\varepsilon>0, p>1$ and $t>0$. There is a weight $w$ : $[-1,1] \rightarrow(0, \infty)$ which satisfies $(3.1)$ but

$$
\int_{0}^{\frac{1}{2}} \log ^{\frac{p-1}{p t}+\varepsilon}(e+1 / w(x)) d x=\infty .
$$

Proof. Clearly we can find $\delta=\delta(p, t, \varepsilon)>0$ small enough such that for $\gamma=\frac{p t}{p-1}-\delta$ and

$$
w(x)=\exp \left(\frac{-1}{|x|^{\gamma}}\right) \frac{1}{|x|^{\gamma+1}} .
$$

we have (4.1). We claim that the weight $w$ satisfies (3.1) with the integrable function $F$ where

$$
F(x)=C|x|^{-\gamma \frac{p-1}{p t}} .
$$


Fix $0 \leq a<b \leq 1$. By the substitution $t=1 / x^{\gamma}$ we see that

$$
\int_{a}^{b} w=-C(\gamma) \int_{1 / a^{\gamma}}^{1 / b^{\gamma}} \exp (-s) d s=C(\gamma)\left(\exp \frac{-1}{b^{\gamma}}-\exp \frac{-1}{a^{\gamma}}\right) .
$$

We want to verify $(3.1)$ for $B=(a, b)$. We need to distinguish two cases.

First suppose that

$$
\exp \frac{-1}{a^{\gamma}}<\frac{1}{2} \exp \frac{-1}{b^{\gamma}} .
$$

Since the function $w(x)$ is increasing for small values of $x$ we conclude from (4.2) and (4.3) that

$$
f_{a}^{b}\left(\frac{w}{f_{a}^{b} w}\right)^{p} \leq C(\gamma, p) f_{a}^{b} \frac{w(x)}{f_{a}^{b} w}\left(\frac{w(x)}{\frac{1}{b-a} \exp \frac{-1}{b^{\gamma}}}\right)^{p-1} d x \leq
$$

$\leq C(\gamma, p) \int_{a}^{b} \frac{w(x)}{f_{a}^{b} w}\left(\frac{\frac{1}{b^{\gamma+1}} \exp \frac{-1}{b^{\gamma}}}{\frac{1}{b-a} \exp \frac{-1}{b^{\gamma}}}\right)^{p-1} d x \leq \frac{C(\gamma, p)}{b^{\gamma(p-1)}} \int_{a}^{b} \frac{w(x)}{f_{a}^{b} w} d x \leq \frac{C(\gamma, p)}{b^{\gamma(p-1)}}$.

The function $F$ is decreasing and therefore

$$
\left(f_{2 B} F\right)^{p t} \geq C(p, t)\left(f_{a}^{b} F\right)^{p t} \geq C(p, t)(F(b))^{p t}=\frac{C(p, t)}{b^{\gamma(p-1)}} .
$$

From (4.4) and (4.5) we obtain (3.1) in this case.

In the second case we have

$$
\exp \frac{-1}{a^{\gamma}}>\frac{1}{2} \exp \frac{-1}{b^{\gamma}}
$$

which implies $a \sim b$. Therefore $w(x) \sim \exp \left(\frac{-1}{b^{\gamma}}\right) \frac{1}{b^{\gamma+1}}$ for every $x \in(a, b)$ which implies

$$
f_{a}^{b}\left(\frac{w}{f_{a}^{b} w}\right)^{p} \leq C(\gamma, p)
$$

Hence (3.1) is clearly valid in this case.

Since (3.1) is satisfied for $B=(a, b)$ if $0 \leq a<b \leq 1$ and everything is symmetric, it is not difficult to verify it also for the general case (i.e. $-1 \leq a<b \leq 1)$.

Example 4.2. Let $\varepsilon>0, p>1$ and $t>0$. There is a weight $w$ : $[-1,1] \rightarrow(0, \infty)$ which satisfies $(3.2)$ but

$$
\int_{0}^{\frac{1}{2}} \log ^{\frac{1}{t}+\varepsilon} \log (e+1 / w(x)) d x=\infty .
$$

Proof. Clearly we can find $\delta>0$ small enough such that for $\gamma=t-\delta$ and

$$
w(x)=\exp \left(-\exp \frac{1}{|x|^{\gamma}}\right) \exp \left(\frac{1}{|x|^{\gamma}}\right) \frac{1}{|x|^{\gamma+1}}
$$

we have (4.6). We claim that (3.2) is satisfied with the integrable function $F(x)=C|x|^{-\gamma / t}$. 
Analogously to the previous example we can show that if $0 \leq a<$ $b \leq 1$ and

$$
\exp \left(-\exp \frac{1}{a^{\gamma}}\right)<\frac{1}{2} \exp \left(-\exp \frac{1}{b^{\gamma}}\right)
$$

then

$$
\begin{gathered}
f_{a}^{b}\left(\frac{w}{f_{a}^{b} w}\right)^{p} \leq C(\gamma, p) f_{a}^{b} \frac{w(x)}{f_{a}^{b} w}\left(\frac{\frac{1}{b^{\gamma+1}} \exp \left(\frac{1}{b^{\gamma}}\right) \exp \left(-\exp \frac{1}{b^{\gamma}}\right)}{\frac{1}{b-a} \exp \left(-\exp \frac{1}{b^{\gamma}}\right)}\right)^{p-1} d x= \\
=\frac{C(\gamma, p)}{b^{(p-1) \gamma}} \exp \left(\frac{p-1}{b^{\gamma}}\right) .
\end{gathered}
$$

The remaining computations are similar to those in Example 4.1.

Example 4.3. Let $\varepsilon>0$ and $\alpha>0$. There is a weight $w:[-1,1] \rightarrow$ $(0, \infty)$ which satisfies $(3.3)$ but

$$
\int_{0}^{\frac{1}{2}} \log ^{\frac{\alpha}{2}+\varepsilon} \log (e+1 / w(x)) d x=\infty .
$$

Proof. Clearly we can find $\delta>0$ small enough such that for $\gamma=\frac{2}{\alpha}-\delta$ and

$$
w(x)=\exp \left(-\exp \frac{1}{|x|^{\gamma}}\right) \exp \left(\frac{1}{|x|^{\gamma}}\right) \frac{1}{|x|^{\gamma+1}}
$$

we have (4.9). We claim that (3.2) is satisfied with the integrable function $F(x)=C|x|^{-\alpha \gamma / 2}$.

If $0 \leq a<b \leq 1$ and (4.7) then

$$
\begin{gathered}
f_{a}^{b} \frac{w}{f_{a}^{b} w} \log ^{\alpha}\left(e+\frac{w}{f_{4 B} F f_{a}^{b} w}\right) \leq \\
\leq C(\gamma, \alpha) f_{a}^{b} \frac{w}{f_{a}^{b} w} \log ^{\alpha}\left(e+\frac{\exp \left(-\exp \frac{1}{b^{\gamma}}\right) \exp \left(\frac{1}{b^{\gamma}}\right) \frac{1}{b^{1+\gamma}}}{\frac{1}{b-a} \exp \left(-\exp \frac{1}{b^{\gamma}}\right)}\right) \leq \frac{C(\gamma, \alpha)}{b^{\alpha \gamma}} .
\end{gathered}
$$

Other details can be carried along the similar lines as in the previous examples.

\section{Applichtions to mappings of finite distortion}

In this section, we give the proofs of Theorem 1.1 and Theorem 1.2. We start with an elementary lemma, which will be used in the proof of Theorem 1.1.

Lemma 5.1. Suppose that $A \subset \mathbb{R}^{n}$ is an open bounded set and that $h: A \rightarrow[1, \infty)$ is a measurable function. Assume that there is $r>1$ and $C_{2}>0$ such that

$$
\int_{A} h(x)^{\gamma} d x \leq \exp \left(C_{2} \gamma^{r}\right)
$$


for every $\gamma \geq 1$. Then there is $s=C(r)>1$ such that

$$
\int_{A} \exp \left(\log ^{s}(h(x))\right)<\infty .
$$

Proof. Let $a \geq 1$ and set $\gamma=a^{\frac{1}{r}}$ and

$$
\Phi_{a}(t)= \begin{cases}\frac{\exp (a)}{\left(\frac{a}{\gamma}\right)^{a}} t & \text { for } t \in\left[0,\left(\frac{a}{\gamma}\right)^{a}\right] \\ \exp \left(\gamma t^{\frac{1}{a}}\right) & \text { for } t \in\left[\left(\frac{a}{\gamma}\right)^{a}, \infty\right) .\end{cases}
$$

It is not difficult to verify that $\Phi_{a}$ is a convex function and that

$$
\Phi_{a}^{-1}(t)= \begin{cases}\frac{\left(\frac{a}{\gamma}\right)^{a}}{\exp a} t & \text { for } t \in[0, \exp (a)] \\ \frac{1}{\gamma^{a}} \log ^{a} t & \text { for } t \in[\exp (a), \infty) .\end{cases}
$$

From Jensen's inequality, (5.2) and (5.1) we obtain

$$
\begin{aligned}
& \Phi_{a}\left(f_{A} \log ^{a} h\right) \leq f_{A} \Phi_{a}\left(\log ^{a} h\right) \leq \frac{1}{|A|}\left(\int_{\left\{\log ^{a} h<\left(\frac{a}{\gamma}\right)^{a}\right\}}+\int_{\left\{\log ^{a} h \geq\left(\frac{a}{\gamma}\right)^{a}\right\}}\right) \leq \\
& \quad \leq \exp a+f_{A} h^{\gamma} \leq \exp a+\frac{1}{|A|} \exp \left(C_{2} \gamma^{r}\right) \leq \exp \left(C\left(C_{2},|A|\right) a\right) .
\end{aligned}
$$

Hence (5.3) gives us

$$
\int_{A} \log ^{a} h \leq C\left(C_{2},|A|\right)^{a} a^{a\left(1-\frac{1}{r}\right)}
$$

for every $a \geq 1$. We set $s=1+\frac{1}{r}$ and compute

$$
\begin{gathered}
\int_{A} \exp \left(\log ^{s}(h(x))\right) d x=\sum_{l=0}^{\infty} \frac{1}{l !} \int_{A} \log ^{s l} h \leq \\
\leq|A|+\sum_{l=1}^{\infty} \frac{1}{l !} C\left(C_{2},|A|, r\right)^{l} l^{l s\left(1-\frac{1}{r}\right)}=|A|+\sum_{l=1}^{\infty} \frac{C^{l} l^{l\left(1-\frac{1}{r^{2}}\right)}}{l !}<\infty .
\end{gathered}
$$

The following lemma gives the doubling property of the Jacobian $J_{f}$ of a mapping $f$ of finite distortion. It is a generalization of $[12$, Theorem 1.5], which gives the doubling property of the Jacobian of a quasiregular mapping. The ideas of the proofs are similar.

Lemma 5.2. Let $f$ be a mapping of finite distortion in $\Omega \subset \mathbb{R}^{n}, n \geq 2$, and suppose that $f$ is continuous, open and discrete and that $f$ satisfies the Lusin condition $N$. Let $n-1<p<n$ and set $t=p /(n-p)$. Let $A \subset \Omega$ be a compact set and assume that the distortion $K(x)$ satisfies $K \in L_{\text {loc }}^{t}(\Omega)$. Then

$$
\int_{2 B} J_{f} \leq C(n, N(f, A)) \exp \left(C(p, n, N(f, A))\left(f_{4 B} K^{t}\right)^{\frac{1}{t(n-1)}}\right) \int_{B} J_{f}
$$


for every ball $B$ such that $4 B \subset A$.

Proof. Fix a ball $B$ centered at $x$ such that $4 B \subset A$. Denote $U_{1}=f(B)$, $U_{2}=f(2 B)$ and $U_{3}=f(4 B)$. Since $f$ is open, $f(x)$ is an interior point of the open connected set $U_{1}$. Set

$$
l=\operatorname{dist}\left(f(x), \partial U_{1}\right) \text { and } L=\max _{y \in \partial U_{2}}|f(x)-y| .
$$

Next, let $E$ be a line segment of length $l$ joining $f(x)$ to $\partial U_{1}$ and let $F$ be a line segment in $\overline{U_{3}} \backslash B(f(x), L)$ joining a point in $\partial U_{2}$ to a point in $\partial U_{3}$. Then $f^{-1}(E)$ joins $x$ to $\partial B$ (use [23, Lemma 2.3] for $B$ ) and $f^{-1}(F)$ joins $\partial 2 B$ to $\partial 4 B$ (use [23, Lemma 2.3] for $4 B$ ).

Since $f$ satisfies the Lusin condition $N$ we obtain from (2.3) and (5.5) that

$$
\int_{B} J_{f} \geq|f(B)| \geq C(n) l^{n} .
$$

Thanks to (2.2), (2.4) and (5.5) we have

$$
\int_{2 B} J_{f} \leq N(f, A)|f(2 B)| \leq C(n) N(f, A) L^{n} .
$$

If $L \leq 2 l$ then (5.6) and (5.7) give us (5.4). In the rest of the proof we will suppose that $L>2 l$ and therefore (2.5) implies that

$$
\operatorname{cap}\left(E, F ; \mathbb{R}^{n}\right) \leq C(n)\left(\log \frac{L}{2 l}\right)^{1-n}
$$

Let $u \in C^{\infty}\left(U_{3}\right)$ be a function such that $u(x) \leq 0$ for $x \in E$ and $u(x) \geq 1$ for $x \in F$. From (2.2) we have that

$$
\int_{4 B}|(\nabla u)(f(x))|^{n} J_{f}(x) d x \leq N(f, A) \int_{\mathbb{R}^{n}}|\nabla u(y)|^{n} d y .
$$

The function $u \circ f$ clearly satisfies all assumptions of Lemma 2.2 for $\tilde{E}=$ $f^{-1}(E)$ and $\tilde{F}=f^{-1}(F)$ and thus we obtain from Hölder's inequality (5.10)

$$
\begin{aligned}
C(p, n) r^{n-p} & \leq \int_{4 B}|\nabla(u \circ f)|^{p} \\
& \leq \int_{4 B}|(\nabla u)(f(x))|^{p}|D f(x)|^{p} d x \\
& \leq\left(\int_{4 B}|(\nabla u)(f(x))|^{n} \frac{|D f(x)|^{n}}{K(x)} d x\right)^{\frac{p}{n}}\left(\int_{4 B} K^{\frac{p}{n-p}}\right)^{\frac{n-p}{n}} \\
& \leq\left(\int_{4 B}|(\nabla u)(f(x))|^{n} J_{f}(x) d x\right)^{\frac{p}{n}}\left(\int_{4 B} K^{\frac{p}{n-p}}\right)^{\frac{n-p}{n}} .
\end{aligned}
$$

Thus taking the infimum of all admissible $u$ we obtain from (5.8), (5.9) and (5.10) that

$$
C(p, n) \leq\left(\frac{1}{r^{n}} \int_{4 B} K^{\frac{p}{n-p}}\right)^{\frac{n-p}{p}} N(f, A)\left(\log \frac{L}{2 l}\right)^{1-n} .
$$


Together with (5.6) and (5.7) this implies (5.4).

The following lemma provides us with a reverse inequality for the Jacobian $J_{f}$ of a mapping $f$ with finite distortion, from which we can deduce the integrability result on $1 / J_{f}$ by the results in Section 3 .

Lemma 5.3. Let $f$ be a mapping of finite distortion in $\Omega \subset \mathbb{R}^{n}, n \geq 2$ and let $A \subset \Omega$ be a compact set. Suppose that $f$ satisfies all assumptions of Lemma 5.2 and that the distributional Jacobian of $f$ is equal to the pointwise Jacobian of $f$. Let $n-1<p<n$ and set $t=p /(n-p)$. Assume that the distortion $K(x)$ satisfies $K^{t} \in L_{\mathrm{loc}}^{1}(\Omega)$. Then

$$
\begin{aligned}
f_{B} J_{f} \leq & C(n, N(f, A)) \exp \left(C(p, n, N(f, A))\left(f_{2 B} K^{t}\right)^{\frac{1}{t(n-1)}}\right) . \\
& \cdot\left(f_{B} K^{t}\right)^{\frac{1}{t}}\left(f_{B} J_{f}^{\frac{n t-t}{n t-n+1}}\right)^{\frac{n t-n+1}{n t-t}}
\end{aligned}
$$

for every ball $B$ such that $2 B \subset A$.

Proof. Fix a ball $B=B\left(x_{0}, s\right)$ such that $B_{2 s}:=B\left(x_{0}, 2 s\right) \subset A$. Under our assumptions on the mapping $f$, we have the following integral form of the isoperimetric inequality, namely,

$$
\int_{B_{r}} J_{f} d x \leq C(n)\left(\int_{\partial B_{r}}|D f|^{n-1} d x\right)^{\frac{n}{n-1}}
$$

for almost every radius $r<s$, where $B_{r}$ is the ball with radius $r$ and the same center as $B_{s}$, see [22]. We raise both sides of inequality (5.11) to the power $(n-1) / n$, and integrate with respect to $r$ over $(0, s)$ to obtain that

$$
\frac{s}{2}\left(\int_{B_{s} / 2} J_{f} d x\right)^{\frac{n-1}{n}} \leq \int_{0}^{s}\left(\int_{B_{r}} J_{f} d x\right)^{\frac{n-1}{n}} d s \leq C(n) \int_{B_{s}}|D f|^{n-1} d x .
$$

We estimate the right-hand side of (5.12) by the distortion inequality and Hölder's inequality and arrive at

$$
\begin{aligned}
\int_{B_{s}}|D f|^{n-1} d x & \leq \int_{B_{s}} K(x)^{\frac{n-1}{n}} J_{f}^{\frac{n-1}{n}} d x \\
& \leq\left(\int_{B_{s}} K(x)^{t} d x\right)^{\frac{n-1}{n t}}\left(\int_{B_{s}} J_{f}^{\frac{n t-t}{n t-n+1}} d x\right)^{\frac{n t-n+1}{n t}} .
\end{aligned}
$$

Thus, we obtain by combining (5.12) and (5.13) that

$$
f_{B_{s / 2}} J_{f} d x \leq C(n)\left(f_{B} K^{t}\right)^{\frac{1}{t}}\left(f_{B} J_{f}^{\frac{n t-t}{n t-n+1}}\right)^{\frac{n t-n+1}{n t-t}} .
$$

The lemma follows from the above inequality and Lemma 5.2. 
Proof of Theorem 1.2. Under the assumptions $f \in W_{\text {loc }}^{1, n}$ and $K \in$ $L_{\text {loc }}^{n-1+\varepsilon}$ it is known that the mapping $f$ of finite distortion is continuous, discrete and open and satisfies the condition $N$ and that distributional Jacobian of $f$ is equal to the pointwise Jacobian of $f$ (see [10], [18], [28], [24], [25] and [20]).

Let $p, n-1<p<n$, be the number such that $t=p /(n-p)=$ $n-1+\varepsilon$. We may use Lemma 5.3 to obtain

$$
f_{B} J_{f} \leq \exp \left(C(\varepsilon, n, N(f, A))\left(f_{2 B} K^{t}\right)^{\frac{1}{t(n-1)}}\right)\left(f_{B} J_{f}^{\frac{n t-t}{n t-n+1}}\right)^{\frac{n t-n+1}{n t-t}}
$$

for every ball $B$ such that $2 B \subset A$. Now we may apply Theorem 3.7 to conclude the proof of Theorem 1.2.

The assumptions of Lemma 5.3 are satisfied also for $f$ in a SobolevOrlicz space which is slightly larger than $W^{1, n}$ (see [26] and references given there for details) and therefore it is possible to obtain the result of Theorem 1.2 also under this weaker condition.

Proof of Theorem 1.1. Suppose $f$ is a mapping with exponentially integrable distortion. Again the known results (see [24], [25], [16], [17], [20]) tell us that the assumptions of Lemma 5.3 on $f$ are satisfied. Pick $p$ such that $n-1<p<n$ and

$$
\tilde{p}:=\frac{n t-n+1}{n t-t}=\frac{n+1}{n+1 / 2},
$$

where $t=p /(n-p)$. We claim that for every $C_{1}>0$ and for every ball $\tilde{B} \subset \subset \Omega$ we have

$$
\exp \left(C_{1}\left(f_{\tilde{B}} K^{t}\right)^{\frac{1}{t(n-1)}}\right) \leq f_{\tilde{B}}\left(C(n)+\exp \left(C_{1} K^{\frac{1}{n-1}}\right)\right) .
$$

Indeed, there are $C_{3}=C(n) \geq 0$ and $C_{4}=C(n) \geq 0$ such that the function

$$
\Phi(y)= \begin{cases}C_{4} y & \text { for } y \in\left[0, C_{3}\right] \\ \exp \left(y^{\left.\frac{1}{t(n-1)}\right)}\right) & \text { for } y>C_{3}\end{cases}
$$

is continuous and convex and clearly

$$
\exp \left(C_{1}\left(f_{\tilde{B}} K^{t}\right)^{\frac{1}{t(n-1)}}\right) \leq \Phi\left(C_{3}\right)+\Phi\left(C_{1}^{t(n-1)} f_{\tilde{B}} K^{t}\right) .
$$

From Jensen's inequality we now obtain

$$
\begin{gathered}
\Phi\left(C_{1}^{t(n-1)} f_{\tilde{B}} K^{t}\right) \leq f_{\tilde{B}} \Phi\left(C_{1}^{t(n-1)} K^{t}\right) \leq \\
\leq f_{\tilde{B}}\left(\Phi\left(C_{3}\right)+\exp \left(C_{1} K^{\frac{1}{n-1}}\right)\right) .
\end{gathered}
$$

From (5.15) and (5.16) we deduce (5.14). 
Let $\gamma>0$ and find $\tilde{t}>0$ such that $\gamma=\frac{2}{3} \frac{\tilde{p}-1}{\tilde{p} \tilde{t}}$ (note that $\tilde{p}=C(n)>$ 1). From Lemma 5.3, elementary inequality and (5.14) we obtain that

$$
\begin{aligned}
f_{B} J_{f} \leq & C(n, N(f, A)) \exp \left(C(n, N(f, A))\left(f_{2 B} K^{t}\right)^{\frac{1}{t(n-1)}}\right) . \\
& \cdot\left(f_{B} K^{t}\right)^{\frac{1}{t}}\left(f_{B} J_{f}^{\frac{1}{\tilde{p}}}\right)^{\tilde{p}} \\
\leq & \left(\exp \left(\frac{C(n, N(f, A))}{\tilde{p} \tilde{t}}\left(f_{2 B} K^{t}\right)^{\frac{1}{t(n-1)}}\right)\right)^{\tilde{p} \tilde{t}}\left(f_{B} J_{f}^{\frac{1}{\tilde{p}}}\right)^{\tilde{p}} \\
\leq & \left(f_{2 B}\left(C(n)+\exp \left(\frac{C(n, N(f, A))}{\tilde{p} \tilde{t}} K^{\frac{1}{n-1}}\right)\right)\right)^{\tilde{p} \tilde{t}}\left(f_{B} J_{f}^{\frac{1}{\tilde{p}}}\right)^{\tilde{p}}
\end{aligned}
$$

for every ball $B$ such that $2 B \subset A$. For $n=2$ we set $\beta=\frac{C(n, N(f, A))}{\tilde{p} \tilde{t}}$ and from (5.17) and Theorem 3.5 for $w=J_{f}^{1 / \tilde{p}}$, and $\varepsilon=\frac{\tilde{p}-1}{3 \tilde{p} \tilde{t}}$ we conclude (1.4).

Further let $n \geq 3, \beta>0$ and $\gamma \geq 1$ so that $\tilde{t} \leq 1$. As before we obtain from (5.17), Theorem 3.5 and Remark 3.6 that

$$
\begin{aligned}
& \int_{B} \log ^{\frac{2}{3}} \frac{\tilde{p}-1}{\tilde{p} \tilde{t}}\left(e+\frac{1}{J_{f}^{\frac{1}{\tilde{p}}}(x)}\right) d x \leq \\
& \quad \leq C\left(\tilde{p}, n,|B|, \int_{B} J_{f}^{\frac{1}{\tilde{p}}}\right)^{\frac{1}{\tilde{t}}} \int_{2 B}\left(C(n)+\exp \left(\frac{C(n, N(f, A))}{\tilde{p} \tilde{t}} K^{\frac{1}{n-1}}\right)\right)
\end{aligned}
$$

for every ball such that $2 B \subset A$. Thanks to the elementary inequality

$$
\begin{gathered}
\int_{2 B} \exp \left(C_{1} \gamma K^{\frac{1}{n-1}}\right) \leq \int_{\left\{C_{1} \gamma K^{\frac{1}{n-1}}<\beta K\right\}}+\int_{\left\{C_{1} \gamma K^{\frac{1}{n-1}} \geq \beta K\right\}} \leq \\
\leq \int_{2 B}\left(\exp (\beta K)+\exp \left(C\left(C_{1}, \beta, n\right) \gamma^{1+\frac{1}{n-2}}\right)\right)
\end{gathered}
$$

we obtain from (5.18), $\tilde{p}=C(n)$ and $\tilde{t}=\frac{C(n)}{\gamma}$ that

$$
\begin{gathered}
\int_{B} \log ^{\gamma}\left(e+\frac{1}{J_{f}(x)}\right) d x \leq \exp \left(C_{2} \gamma^{1+\frac{1}{n-2}}\right) \text { for every } \gamma \geq 1 \text { and } 2 B \subset A \\
\text { where } C_{2}=C\left(n, \beta, N(f, A),|B|, \int_{B} J_{f}^{\frac{1}{\tilde{p}}}, \int_{2 B} \exp (\beta K)\right) .
\end{gathered}
$$

Therefore our conclusion (1.3) follows from Lemma 5.1.

\section{EXAMPLES OF MAPPINGS OF FINITE DISTORTION}

The following lemma can be verified by an elementary calculation.

Lemma 6.1. Let $\rho:(0, \infty) \rightarrow(0, \infty)$ be a strictly monotone, differentiable function. Then for the mapping

$$
f(x)=\frac{x}{\|x\|} \rho(\|x\|), \quad x \neq 0
$$


we have for almost every $x$

and

$$
D f(x) \sim \max \left\{\frac{\rho(\|x\|)}{\|x\|},\left|\rho^{\prime}(\|x\|)\right|\right\}
$$

$$
J_{f}(x) \sim \frac{\rho^{\prime}(\| x||) \rho(\|x\|)^{n-1}}{\|x\|^{n-1}} .
$$

Our first example shows us that under the integrability of a power of $K$ we cannot expect for a better result than

$$
\log ^{\gamma}\left(e+\frac{1}{J_{f}}\right) \in L^{1}
$$

for some $\gamma>0$. The positive result from Theorem 1.2 is weaker than this.

Example 6.2. Let $t>0$. There is a mapping of finite distortion $f: B(0,2) \rightarrow \mathbb{R}^{n}$ such that $f$ is Lipschitz continuous, for every $\varepsilon>0$ we have

$$
\begin{gathered}
K \in L^{\frac{t}{n-1}-\varepsilon}(B(0,2)) \text { and } \log ^{t-\varepsilon}\left(e+\frac{1}{J_{f}(x)}\right) d x \in L^{1}(B(0,2)), \\
\text { but } \int_{B(0,1)} \log ^{t}\left(e+\frac{1}{J_{f}(x)}\right) d x=\infty .
\end{gathered}
$$

Proof. Set

$$
\begin{gathered}
\alpha=\frac{n}{t}, h(t)=\exp \left(-\frac{1}{t^{\alpha}}\right) \text { and } \\
f(x)= \begin{cases}\frac{x}{\|x\|} h(\|x\|) & \text { for } x \neq 0 \\
0 & \text { for } x=0 .\end{cases}
\end{gathered}
$$

From Lemma 6.1 we see that

$$
|D f(x)| \sim \exp \left(-\frac{1}{\|x\|^{\alpha}}\right) \frac{1}{\|x\|^{\alpha+1}} \text { for } x \neq 0
$$

and therefore $f$ is Lipschitz continuous thanks to $\lim _{x \rightarrow 0}|D f(x)|=0$. Analogously we obtain

$$
J_{f}(x) \sim \exp \left(-\frac{n}{\|x\|^{\alpha}}\right) \frac{1}{\|x\|^{n+\alpha}} \text { and thus } K(x) \sim \frac{1}{\|x\|^{(n-1) \alpha}}
$$

for every $x \neq 0$. Conditions (6.1) and (6.2) can be now easily verified by switching to polar coordinates.

Our second example tells us that, based on the integrability of $\exp (\beta K)$, one cannot expect for a better result than

$$
\exp \left(C \log ^{\frac{n-1}{n}}\left(e+\frac{1}{J_{f}(x)}\right)\right) \in L^{1}
$$

for some constant $C>0$. Again the positive result obtained from Theorem 1.1 is not optimal. 
Example 6.3. There is a mapping $f$ of finite distortion defined in $B(0,1 / 2)$ and constants $c_{1}, c_{2}, c_{3}>0$ such that $f$ is Lipschitz continuous

$$
\begin{gathered}
\exp \left(c_{1} K\right) \in L^{1}\left(B\left(0, \frac{1}{2}\right)\right) \text { and } \\
\exp \left(c_{2} \log ^{\frac{n-1}{n}}\left(e+\frac{1}{J_{f}(x)}\right)\right) \in L^{1}\left(B\left(0, \frac{1}{2}\right)\right), \\
\text { but } \int_{B\left(0, \frac{1}{4}\right)} \exp \left(c_{3} \log ^{\frac{n-1}{n}}\left(e+\frac{1}{J_{f}(x)}\right)\right) d x=\infty .
\end{gathered}
$$

Proof. Set

$$
\begin{gathered}
a=\frac{n}{n-1}, h(t)=\exp \left(-|\log t|^{a}\right) \text { and } \\
f(x)= \begin{cases}\frac{x}{\|x\|} h(\|x\|) & \text { for } x \neq 0 \\
0 & \text { for } x=0 .\end{cases}
\end{gathered}
$$

From Lemma 6.1 we have that

$$
|D f(x)| \sim \exp \left(-\left.|\log \| x|\right|^{a}\right) \frac{\left.|\log \| x|\right|^{a-1}}{\|x\|} \text { for } x \neq 0
$$

and therefore $f$ is Lipschitz continuous in view of $\lim _{x \rightarrow 0}|D f(x)|=0$. Analogously we see that

$$
\begin{aligned}
& \qquad J_{f}(x) \sim \exp \left(-\left.n|\log \| x|\right|^{a}\right) \frac{|\log \|x\||^{a-1}}{\|\left. x\right|^{n}} \\
& \text { and thus } K(x) \sim|\log \|x\||^{(a-1)(n-1)}=-\log \|x\|
\end{aligned}
$$

for every $x \neq 0$. Since $\left(\log \frac{1}{J_{f}(x)}\right)^{\frac{n-1}{n}} \sim-\log \|x\|$ it is not difficult to verify conditions (6.3) and (6.4).

\section{REFERENCES}

[1] K. Astala, T. Iwaniec, P. Koskela and G. Martin, Mappings of BMO-bounded distortion, Math. Ann. 317 no. 4 (2000), 703-726.

[2] S. M. Buckley, Estimates for operator norms on weighted spaces and reverse Jensen inequalities, Trans. Amer. Math. Soc. 340 no. 1 (1993), 253-272.

[3] R. Coifman and C. Fefferman, Weighted norm inequalities for maximal functions and singular integrals, Studia Math. 51 (1974), 241-250.

[4] G. David, Solutions de l'equation de Beltrami avec $\|\mu\|=1$, Ann. Acad. Sci. Fenn. Ser. A I Math. 13 no. 1 (1988), 25-70.

[5] A. Elcrat and N. Meyers, Some results on the regularity for solutions of nonlinear elliptic systems and quasiregular functions, Duke Math. J. 42 (1975), 121-136.

[6] D. Faraco, P. Koskela and X. Zhong, Mappings of finite distortion: The degree of regularity, Adv. Math. 90 (2005), 300-318.

[7] H. Federer, Surface area II., Trans. Amer. Math. Soc. 55 (1944), 438-456.

[8] R. Fefferman, A criterion for the absolute continuity of the harmonic measure associated with an elliptic operator, J. Amer. Math. Soc. 2 no. 1 (1989), 127-135. 
[9] F. W. Gehring, The $L^{p}$-integrability of the partial derivatives of a quasiconformal mapping, Acta Math. 130 (1973), 265-277.

[10] V. Gol'dstein and S. Vodop'yanov, Quasiconformal mappings and spaces of functions with generalized first derivatives, Sibirsk. Mat. Z. 17 (1976), 515531.

[11] J. Heinonen and P. Koskela, Sobolev mappings with integrable dilatations, Arch. Rational Mech. Anal. 125 no. 1 (1993), 81-97.

[12] J. Heinonen and P. Koskela, Weighted Sobolev and Poincaré inequalities and quasiregular mappings of polynomial type, Math. Scand. 77 (1995), 251-271.

[13] J. Heinonen and P. Koskela, Quasiconformal maps in metric spaces with controlled geometry, Acta Math. 181 (1998), 1-61.

[14] S. Hencl and J. Malý, Mappings of finite distortion: Hausdorff measure of zero sets, Math. Ann. 324 (2002), 451-464.

[15] D. A. Herron and P. Koskela, Conformal capacity and the quasihyperbolic metric, Indiana Univ. Math. J. 45 no. 2 (1996), 333-359.

[16] T. Iwaniec, P. Koskela and G. Martin, Mappings of BMO-distortion and Beltrami type operators, J. Analyse Math. 88 (2002), 337-381.

[17] T. Iwaniec, P. Koskela, G. Martin and C. Sbordone, Mappings of exponentially integrable distortion, J. London Math. Soc. 67 no. 1 (2003), 123-136.

[18] T. Iwaniec, P. Koskela and J. Onninen, Mappings of finite distortion: Monotonicity and continuity, Invent. Math. 144 (2001), 507-531.

[19] T. Iwaniec and G. Martin, Geometric function theory and nonlinear analysis, Oxford Mathematical Monographs, The Clarendon Press, Oxford University Press, New York, 2001.

[20] T. Iwaniec and C. Sbordone, On the integrability of the Jacobian under minimal hypotheses, Arch. Rational Mech. Anal. 119 no. 2 (1992), 129-143.

[21] T. Iwaniec and V. Šverák, On mappings with integrable dilatation, Proc. Amer. Math. Soc. 118 (1993), 181-188.

[22] J. Onninen, A note on the isoperimetric inequality, Proc. Amer. Math. Soc. 131 no. 12 (2003), 3821-3825.

[23] S. Kallunki, Mappings of finite distortion: The metric definition, Ann. Acad. Sci. Fenn. Math. Diss. 131 (2002), 1-33.

[24] J. Kauhanen, P. Koskela and J. Malý, Mappings of finite distortion: Discreteness and openness, Arch. Ration. Mech. Anal. 160 (2001), 135-151.

[25] J. Kauhanen, P. Koskela and J. Malý, Mappings of finite distortion: Condition N, Michigan Math. J. 49 (2001), 169-181.

[26] J. Kauhanen, P. Koskela, J. Malý, J. Onninen and X. Zhong, Mappings of finite distortion: Sharp Orlicz-conditions, Rev. Mat. Iberoamericana 19 (2003), 857872.

[27] P. Koskela and J. Malý, Mappings of finite distortion: the zero set of the Jacobian, J. Eur. Math. Soc. 5 no. 2 (2003), 95-105.

[28] J. Manfredi and E. Villamor, An extension of Reshetnyak's theorem, Indiana Univ. Math. J. 47 no. 3 (1998), 1131-1145.

[29] O. Martio, On the integrability of the derivate of a quasiregular mapping, Math. Scand. 35 (1974), 43-48.

[30] B. Muckenhoupt, Weighted norm inequalities for the Hardy maximal function, Trans. Amer. Math. Soc. 165 (1972), 207-226.

[31] Yu. G. Reshetnyak, Space Mappings with Bounded Distortion, Trans. of Mathematical Monographs, Amer. Math. Soc, vol. 73, 1989.

[32] S. Rickman, Quasiregular mappings, Ergebnisse der Mathematik und ihrer Grenzgebiete (3) [Results in Mathematics and Related Areas (3)], 26. SpringerVerlag, Berlin, 1993. 
Department of Mathematics And Statistics, University OF JyVÄSKYlä, P.O. Box 35 (MAD), FIN-40014 University of JyvÄSKylä, Finland E-mail address: hencl@maths.jyu.fi

E-mail address: pkoskela@maths.jyu.fi

E-mail address: zhong@maths.jyu.fi 